\title{
A FIRST COMPARISON OF KEPLER PLANET CANDIDATES IN SINGLE AND MULTIPLE SYSTEMS
}

\author{
David W. Latham ${ }^{1}$, Jason F. Rowe ${ }^{2}$, Samuel N. Quinn ${ }^{1}$, Natalie M. Batalha ${ }^{3}$, William J. Borucki ${ }^{2}$, \\ Timothy M. Brown ${ }^{4}$, Stephen T. Bryson ${ }^{2}$, Lars A. Buchhave ${ }^{5}$, Douglas A. Caldwell ${ }^{6}$, Joshua A. Carter ${ }^{1,20}$, \\ Jessie L. Christiansen ${ }^{6}$, David R. Ciardi ${ }^{7}$, William D. Cochran ${ }^{8}$, Edward W. Dunham ${ }^{9}$, Daniel C. Fabrycky ${ }^{10,20}$, \\ Eric B. Ford ${ }^{11}$, Thomas N. Gautier, III ${ }^{12}$, Ronald L. Gilliland ${ }^{13}$, Matthew J. Holman ${ }^{1}$, Steve B. Howell ${ }^{2,14}$, \\ Khadeejah A. Ibrahim ${ }^{15}$, Howard Isaacson ${ }^{16}$, Jon M. Jenkins ${ }^{6}$, David G. Koch $^{2}$, Jack J. Lissauer ${ }^{2}$, Geoffrey W. Marcy ${ }^{16}$, \\ Elisa V. Quintana ${ }^{6}$, Darin Ragozzine ${ }^{1}$, Dimitar Sasselov ${ }^{1}$, Avi ShPorer ${ }^{4,17}$, Jason H. Steffen ${ }^{18}$, William F. Welsh ${ }^{19}$, \\ AND BILl WOHLER ${ }^{15}$ \\ ${ }^{1}$ Harvard-Smithsonian Center for Astrophysics, 60 Garden Street, Cambridge, MA 02138, USA \\ ${ }^{2}$ NASA Ames Research Center, Moffett Field, CA 94035, USA \\ ${ }^{3}$ Department of Physics and Astronomy, San Jose State University, San Jose, CA 95192, USA \\ ${ }^{4}$ Las Cumbres Observatory Global Telescope, Goleta, CA 93117, USA \\ ${ }^{5}$ Niels Bohr Institute, Copenhagen University, DK-2100 Copenhagen, Denmark \\ ${ }^{6}$ SETI Institute/NASA Ames Research Center, Moffett Field, CA 94035, USA \\ ${ }^{7}$ NASA Exoplanet Science Institute/Caltech, Pasadena, CA 91125, USA \\ ${ }^{8}$ Department of Astronomy, University of Texas, Austin, TX 78712, USA \\ ${ }^{9}$ Lowell Observatory, Flagstaff, AZ 86001, USA \\ ${ }^{10}$ Department of Astronomy and Astrophysics, University of California, Santa Cruz, CA 95064, USA \\ ${ }^{11}$ Department of Astronomy, University of Florida, Gainesville, FL 32111, USA \\ 12 Jet Propulsion Laboratory/California Institute of Technology, Pasadena, CA 91109, USA \\ ${ }^{13}$ Space Telescope Science Institute, Baltimore, MD 21218, USA \\ ${ }^{14}$ National Optical Astronomy Observatory, Tucson, AZ 85719, USA \\ 15 Orbital Sciences Corporation/NASA Ames Research Center, Moffett Field, CA 94035, USA \\ ${ }^{16}$ Department of Astronomy, University of California, Berkeley, CA 94720, USA \\ ${ }^{17}$ Department of Physics, University of California, Santa Barbara, CA 93106, USA \\ ${ }^{18}$ Fermilab Center for Particle Astrophysics, P.O. Box 500, Batavia, IL 60510, USA \\ ${ }^{19}$ Department of Astronomy, San Diego State University, San Diego, CA 92182, USA \\ Received 2011 March 9; accepted 2011 March 21; published 2011 April 15
}

\begin{abstract}
In this Letter, we present an overview of the rich population of systems with multiple candidate transiting planets found in the first four months of Kepler data. The census of multiples includes 115 targets that show two candidate planets, 45 with three, eight with four, and one each with five and six, for a total of 170 systems with 408 candidates. When compared to the 827 systems with only one candidate, the multiples account for $17 \%$ of the total number of systems, and one-third of all the planet candidates. We compare the characteristics of candidates found in multiples with those found in singles. False positives due to eclipsing binaries are much less common for the multiples, as expected. Singles and multiples are both dominated by planets smaller than Neptune; $69_{-3}^{+2} \%$ for singles and $86_{-5}^{+2} \%$ for multiples. This result, that systems with multiple transiting planets are less likely to include a transiting giant planet, suggests that close-in giant planets tend to disrupt the orbital inclinations of small planets in flat systems, or maybe even prevent the formation of such systems in the first place.
\end{abstract}

Key words: planetary systems

\section{INTRODUCTION}

Although it was anticipated that NASA's Kepler mission could find systems with more than one planet transiting the same host star (Koch \& Borucki 1996; Holman \& Murray 2005), the rich harvest of candidate multiples that appeared already in the first four months of Kepler data caught all of us on the Kepler Science Team by surprise. The first announcement of five multiples (Steffen et al. 2010) was timed to coincide with the initial public data release on 2010 June 15 (Borucki et al. 2011a). Systems with multiple transiting planets are rich with information that provides additional constraints on the characteristics of the planets and even their host stars (Ragozzine \& Holman 2011), as illustrated by two examples of multiple planet systems exhibiting transit time variations that constrain the masses of the planets: Kepler-9 with three transiting planets (Holman et al. 2010; Torres et al. 2011), and

\footnotetext{
${ }^{20}$ Hubble Fellow.
}

Kepler-11 with six (Lissauer et al. 2011a). In this Letter, we present an overview of the full population of multiples that show transits in the first four months of Kepler data. Note that we have not attempted to correct for the probability that planetary orbits are properly aligned to show transits, or for the dependence of transit detectability on various noise sources. Instead we have chosen to compare singles with multiples in ways that should minimize these biases.

\section{KEPLER OBJECTS OF INTEREST}

Kepler targets that show features in their light curves that might be due to transits are designated "Kepler Objects of Interest" (KOIs). The KOI numbering convention is that the digits before the decimal point specify a unique target, and the two digits after specify a planet candidate, in the order that it was identified for that target. There is no simple description of how KOIs were identified because the procedures evolved considerably as the data improved and the team gained experience. The general approach used for the identification of KOIs is described 
by Borucki et al. (2011b). Here we present some additional details, with special emphasis on the procedures that were used to identify candidates in multiples.

Initially, KOIs were identified by visual inspection of light curves for candidates identified by the Kepler pipeline using the Transiting Planet Search (TPS; Jenkins et al. 2010) on individual quarters of data. The Data Validation (DV; Wu et al. 2010) reports from the pipeline were then used to identify false positives involving centroid motion during dimmings and also to identify additional candidates. This effort resulted in nearly 1000 KOIs and somewhat less than 100 systems of multiple candidates.

The next major release of the Kepler pipeline will stitch quarters together, so that TPS and DV can work on light curves from multiple quarters. As a stopgap, a stand-alone tool for analyzing multiple quarters was developed by Jason Rowe. Starting with the calibrated (raw) time series, sections were excised that showed instrumental artifacts, such as gaps due to safe modes of the spacecraft and subsequent thermal settling. The light curves were next detrended with a high-pass filter (to reduce sensitivity to instrumental drifts and long-term stellar variability) and then were searched for transits using a version of the Box Least Squares (BLS; Kovács et al. 2002) algorithm. Multiquarter data for nearly 180,000 targets were searched for transits (some targets were observed for only one or two quarters). Any event that was detected above a $3 \sigma$ threshold was sent to routines that attempted to fit a planetary-transit model, adopting the stellar parameters $\left(T_{\text {eff }}, \log g\right.$, and $\left.R_{\star}\right)$ from the Kepler Input Catalog (KIC; Brown et al. 2011). Plots of the successful fits, about 25,000 in all, were then inspected visually. This effort led to about 600 additional KOIs, with nearly 100 of them in multiples.

The stopgap multiquarter pipeline was then run again on the earlier set of KOIs, after removing the sections of the light curves affected by the previously identified transits, to look for additional candidates. This process was iterated until no more candidates were found. This effort identified more than 100 new candidates in multiple systems, in addition to the candidates that had been identified previously using the quarter-by-quarter analysis.

\section{FALSE POSITIVES}

KOIs are reviewed from time to time by the Kepler team, to determine which ones should be prioritized for additional follow-up observations of various types, and which ones are likely false positives that can be retired to the inactive list. In the paper summarizing the characteristics of the planet candidates identified in the first four months of Kepler data, Borucki et al. (2011b) present a list in their Table 4 of 498 KOIs that had been identified as false positives and were no longer considered to be viable planet candidates. In Table 1, we summarize the number of false positives compared to the number of surviving candidates among the KOIs, with a separate accounting for the singles and multiples.

More than half of the false positives $(59 \%)$ resulted from an "active pixel offset" (APO). This test uses a difference image analysis to show that during transit-like events the image is significantly displaced from the target position and is star-like, indicating contamination by a faint background eclipsing binary or by the wings of the point spread function from a nearby bright star encroaching on the edge of the target aperture. Most of the remaining false positives also involved eclipsing binaries, and were identified either by features in the Kepler light curves,
Table 1

Census of False Positives

\begin{tabular}{|c|c|c|}
\hline Number & Description & Fraction \\
\hline 1733 & KOIs identified for 1489 targets & \\
\hline 1235 & Survivor KOIs for 997 targets & $1235 / 1733=0.713$ \\
\hline 827 & Survivors in singles & $827 / 1235=0.670$ \\
\hline \multirow[t]{7}{*}{408} & Survivors in multiples & $408 / 1235=0.330$ \\
\hline & 170 multiple systems & $170 / 997=0.171$ \\
\hline & 115 systems with 2 KOIs & $230 / 408=0.563$ \\
\hline & 45 systems with 3 KOIs & $135 / 408=0.330$ \\
\hline & 8 systems with 4 KOIs & $32 / 408=0.078$ \\
\hline & 1 system with 5 KOIs & $5 / 408=0.012$ \\
\hline & 1 system with 6 KOIs & $6 / 408=0.015$ \\
\hline 498 & False positives & $498 / 1733=0.288$ \\
\hline \multirow[t]{2}{*}{486} & False positives in singles & $486 / 498=0.976$ \\
\hline & Rate per KOIs in singles & $486 /(486+827)=0.370$ \\
\hline 288 & Active pixel offsets (APO) & $288 / 486=0.592$ \\
\hline 164 & Light curve evidence & $164 / 486=0.337$ \\
\hline 23 & Spectroscopic binaries & $23 / 486=0.047$ \\
\hline 11 & Photometric false alarms & $11 / 486=0.023$ \\
\hline \multirow[t]{2}{*}{12} & False positives in 6 systems & $12 / 498=0.024$ \\
\hline & Rate per KOIs in multiples & $12 / 420=0.029$ \\
\hline 5 & APOs in 3 systems & $3 / 170=0.017$ \\
\hline 5 & Light curve evidence & $5 / 420=0.012$ \\
\hline 2 & In one spectroscopic binary & $1 / 170=0.006$ \\
\hline
\end{tabular}

such as secondary eclipses or ellipsoidal variations or eclipsetime variations $(34 \%)$, or by large variations observed in the radial velocities $(5 \%)$. Eleven of the early KOIs were judged to be photometric false alarms, based on additional data from subsequent quarters.

The difference in the rate of false positives for singles compared to multiples is striking; for the singles the rate is $37 \%$ (486 false positives compared to 827 survivors), but for the multiples the rate is only $3 \%$ (12 false positives in six systems, compared to 408 surviving planets in 170 systems). This large difference is expected, because the APOs for the singles are the result of chance alignments of eclipsing binaries with the full target list of nominally 150,000 stars, while the APOs for the multiples come from chance alignments with systems that already show transit-like events. Lumping together all the false positives among singles due to eclipsing binaries gives a rate of $(288+164+23) / 150,000=0.0032$. Assuming that the KOIs have been drawn from the same parent population as all 150,000 targets, the expected number of doubles involving a planet and a false positive due to an eclipsing binary is roughly $827 \times 0.0032=2.6$, while the number involving two eclipsing binaries and no planets is roughly $486 \times 0.0032=1.5$. The key assumptions here are that the probability that a target image is contaminated by an accidental alignment with a background eclipsing binary is the same, whether the target already shows a transit-like event or not, and that the false-positive probability for a KOI is independent of the depth of the transit-like event in its light curve (multiples mostly show shallower dips). By the same line of argument, the number involving two planets and an eclipsing binary is only $115 \times 0.0032=0.4$. Higher order coincidences are correspondingly less likely. The probability that a double consists of an accidental alignment of two unrelated singles seems less likely than the two eclipsing binary case, because eclipsing binaries are more common than singles in the Kepler sample by a factor of almost three (Prša et al. 2011). These numbers are summarized in Table 1.

These rough estimates of the expected rates of false positives among the multiples are preliminary, because the vetting effort 

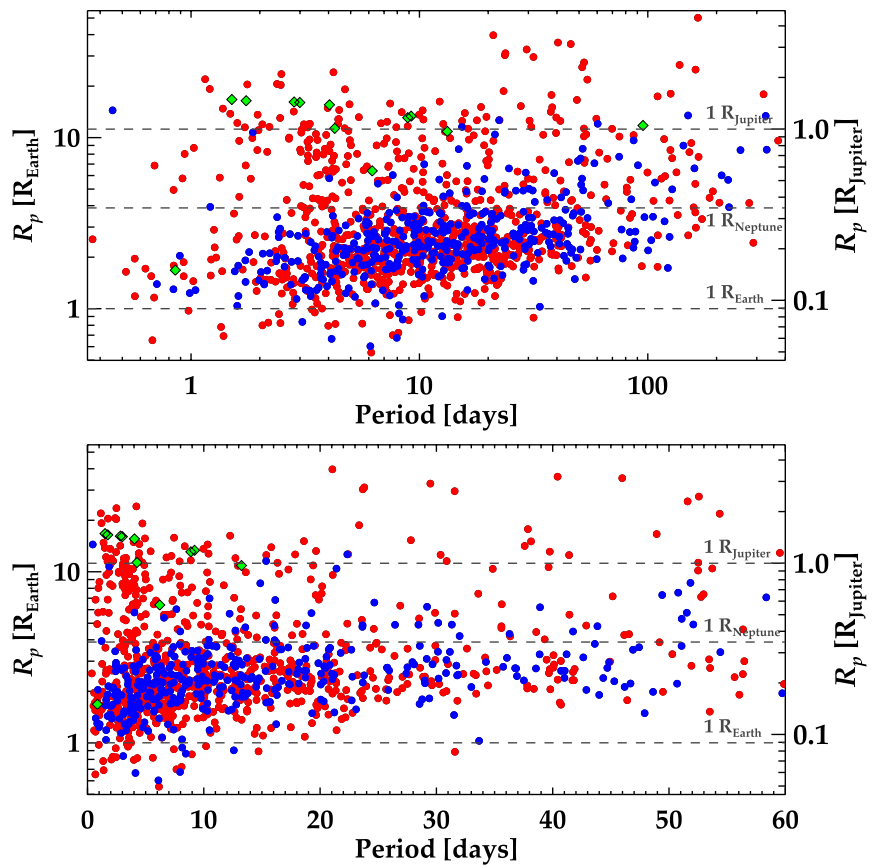

Figure 1. Planet radius vs. orbital period. The bottom panel shows periods shorter than 60 days using a linear scale; the top panel uses a log scale to show the entire range of periods. Planet candidates in singles are plotted in red, and in blue for those in multiples. Kepler finds very few giant planets in systems of multiple planets. This conclusion is not affected by the rather large upper limit that was adopted by Borucki et al. (2011b) for $R_{\mathrm{P}}$. The published CoRoT planets are plotted in green. Only one of these, CoRoT-7b, is smaller than Neptune, and the radial-velocity observations suggest that it may have a non-transiting companion (Queloz et al. 2009).

is still unfinished. Furthermore, some types of false positives, such as hierarchical triples, are extremely difficult to identify, especially for shallow events. Thus, we expect that there are still false positives lurking among both the singles and the multiples. Nevertheless, in general, it must be true that false positives due to chance alignments must be much less common among multiples than singles, and even for singles the rate of residual false positives among the vetted candidates may be as low as $5 \%$ or $10 \%$ (Morton \& Johnson 2011).

\section{PLANET RADIUS VERSUS ORBITAL PERIOD}

The process of fitting transit models to KOI light curves delivers two primary observable characteristics of the candidate planet: the planetary radius, $R_{\mathrm{P}}$ (where we have adopted the KIC value for the stellar radius), and the orbital period, $P$. The plot of these two quantities against each other is shown in Figure 1 where the active KOIs in multiples are blue and the singles are red. Single planets come in all sizes, but there are relatively few giant planets in transiting multiples. The pile-up of giant planets near three days is obvious, with no corresponding pileup of planets, either large or small, among the multiples. The detection limit is especially clear in the lower right corner of the upper panel, where period is logarithmic. The edge of the distribution of detected candidates has a slope of $1 / 3$ as expected (the total number of data points during transits varies nominally as $P^{-2 / 3}$ ).

The distributions of planet radius versus period are shown more quantitatively by the histograms in Figure 2, where we have collapsed Figure 1 onto the $R_{\mathrm{P}}$ and $\log P$ axes in the upper and lower panels, respectively. The vertical scales for the singles and multiples have been normalized so that they both have the
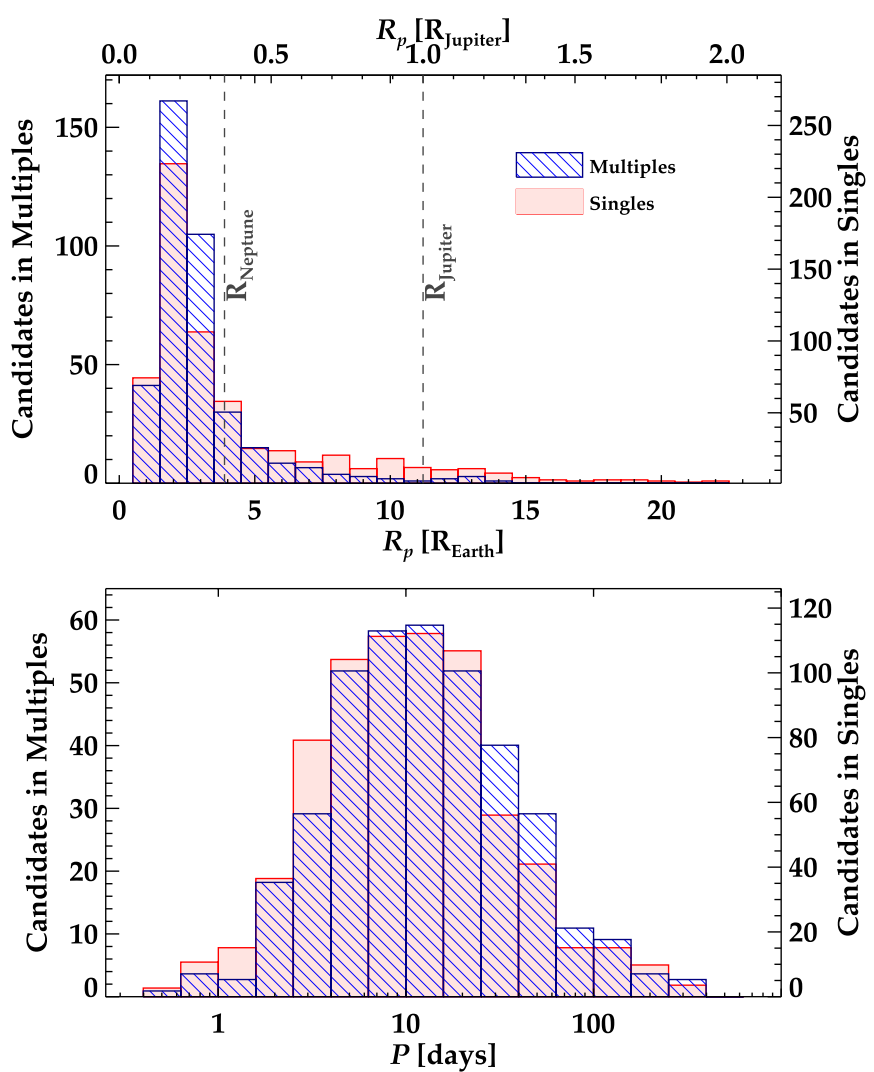

Figure 2. Histograms for the number of planet candidates vs. planetary radius and period. Singles are shown in shaded red, multiples in cross-hatched blue. The vertical scales for the singles and multiples have been normalized so that they both have the same area under their histogram. Planets smaller than Neptune dominate both samples, but more so for the multiples; $69 \%$ for the singles and $86 \%$ for the multiples.

same area under their histograms. Planets smaller than Neptune dominate both samples, but more so for the multiples; $69_{-3}^{+2} \%$ for the singles and $86_{-5}^{+2} \%$ for the multiples. The error estimates for these percentages only consider Poisson noise and do not include any contribution from uncertainties in $R_{\mathrm{P}}$. The difference in the radius distributions between the singles and the multiples is highly significant; the Kolmogorov-Smirnov (K-S) test gives a probability of $2 \times 10^{-10}$ that they are drawn from the same parent distribution.

The period distributions for singles and multiples are quite similar. To the eye there may appear to be a slight shift to shorter periods for the singles, but the significance of this difference is not supported by the K-S test, which reports a probability of $10 \%$ that such differences could occur by chance.

Figure 3 compares the number of singles versus the number of systems that are multiples, as a function of effective temperature. Because nearly all of the host stars are on or near the main sequence, effective temperature is a reasonable proxy for hoststar mass. The K-S test reports that the difference between the two distributions is marginally significant, with a probability of 0.008 and $D$ value of 0.1 . It appears that singles may be more common than multiples around the hotter, more massive stars, while the multiples are more common than singles around the cooler, less massive stars. This might be related to the tendency for close-in giant planets to be less common around low-mass stars (Johnson et al. 2010), and/or to the tendency for small planets in the $R_{\mathrm{P}}$ range 2-4 Earth radii to be much more common around cool stars in the $T_{\text {eff }}$ range $3600-4100 \mathrm{~K}$ 


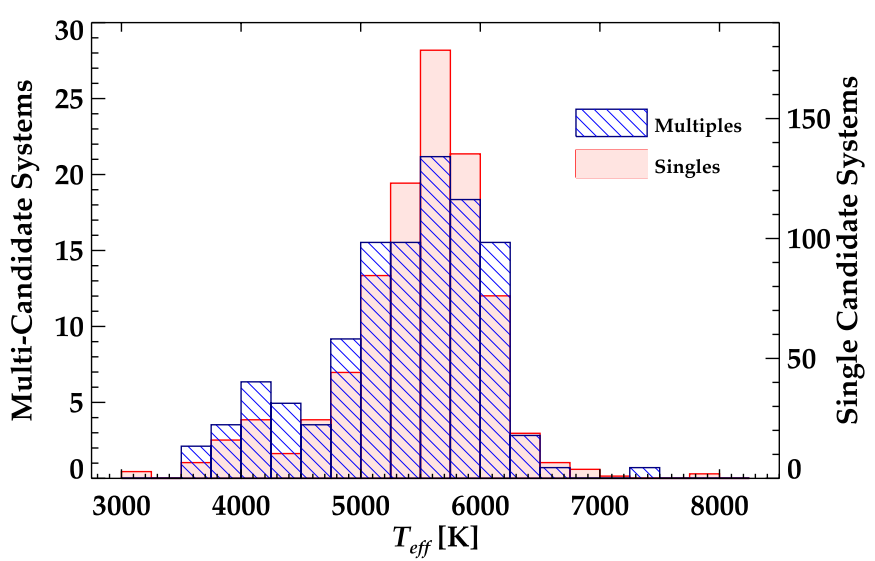

Figure 3. Number of systems vs. effective temperature of the host star, which is a proxy for the stellar mass. Single planets are relatively more common around the hotter stars, multiple planets are more common around the cooler stars. No corrections have been made for the relative number of targets as a function of $T_{\text {eff }}$, or for the probability of detection.

(Howard et al. 2011). Note that this is only a comparison of singles to multiples, and no corrections have been made, either for the relative number of targets as a function of $T_{\text {eff }}$ or for the probability of detection.

\section{DISCUSSION}

The fraction of single planet candidates that are smaller than Neptune is $69_{-3}^{+2} \%(569 / 827)$. The fraction of multiple systems with no planets larger than Neptune is $78_{-7}^{+4} \%(133 / 170)$. Thus, systems with multiple transiting planets are less likely to include a transiting giant planet. If the comparison is restricted to short period planets $(P<10$ days $)$, the difference is particularly striking: the fraction of short-period single candidates that are smaller than Neptune is $69_{-4}^{+3} \%(279 / 405)$, while the fraction of multiple systems that contain at least one short-period planet (117 systems) but no short-period planets larger than Neptune is $96_{-9}^{+2} \%(112 / 117)$. One possible interpretation is that a close-in giant planet can stir up the orbits of other inner planets in its system, while a system of small planets is more likely to preserve the flatness of the disk from which it is formed. This picture is supported by determinations of the spin/orbit alignment for transiting giant planets using the Rossiter-McLaughlin effect. The orbits of some giants are well aligned with the rotation of their host star, while others show significant orbital inclinations, including even retrograde orbits (Winn et al. 2010; Triaud et al. 2010). Thus, there is good evidence that some systems have been disrupted from their presumably flat initial configuration. On the other hand, there is good evidence that close-in giant planets do not always disrupt or prevent the formation of systems with multiple planets. Radial-velocity surveys show that about $25 \%$ of the giant planets are accompanied by companions with smaller minimum masses ${ }^{21}$ (Wright et al. 2009), and the actual fraction may be much higher due to the radial-velocity detection limit for small planets.

We observe the rate of false positives due to eclipsing binaries to be much smaller for multiples than singles. This is expected, because the number of candidates that show a candidate planet or false positive is much smaller than the full list of approximately
150,000 targets. Thus, the probability that an eclipsing binary contaminates the light of a multiple is much smaller than for a single. Lissauer et al. (2011b) present some independent evidence that many of the multiples must be systems of planets, in particular the common occurrence of periods near mean motion resonance. This reinforces the impression that KOIs in multiples are very likely to be planets.

Why has not CoRoT announced any multiples yet? Kepler's better photometric precision and longer time series both contribute to the detection of smaller planets with longer periods, as is needed to discover flat systems. Actually, CoRoT may have come very close to detecting a multiple transiting system, namely CoRoT-7. The transiting planet in this system, CoRoT$7 \mathrm{~b}$, is the smallest discovered so far by $\operatorname{CoRoT}$ (see Figure 1), but the orbit has a rather extreme impact parameter. Thus, additional planets in the system, such as the proposed second planet CoRoT-7c (Queloz et al. 2009), would be less likely to transit also.

Transit time variations for planets in multiple systems promise to be an important tool for constraining the masses of planets that are too small to be detected with current radial-velocity techniques. These constraints improve with longer time series, which is a good argument for extending the Kepler mission and continuing to monitor the most promising multiple systems. This approach may be able to confirm rocky planets in the Habitable Zones of Kepler targets (Ford et al. 2011).

We thank the entire Kepler team for all the hard work that has made these results possible. Funding for this Discovery Mission is provided by NASA's Science Mission Directorate. We give special thanks to the anonymous referee for insightful and timely feedback.

\section{Facility: Kepler}

\section{REFERENCES}

Borucki, W. J., et al. 2011a, ApJ, 728, 117

Borucki, W. J., et al. 2011b, ApJ, submitted (arXiv:1102.0541)

Brown, T. M., Latham, D. W., Everett, M. R., \& Esquerdo, G. A. 2011, AJ, submitted (arXiv:1102.0342)

Ford, E. B., et al. 2011, ApJ, submitted (arXiv:1102.0544)

Holman, M. J., \& Murray, N. W. 2005, Science, 307, 1288

Holman, M. J., et al. 2010, Science, 330, 51

Howard, A. W., et al. 2011, ApJ, submitted (arXiv:1103.2541)

Jenkins, J. M., et al. 2010, Proc. SPIE, 7740, 10

Johnson, J. A., Aller, K. M., Howard, A. W., \& Crepp, J. R. 2010, PASP, 122 905

Koch, D., \& Borucki, W. 1996, in The First International Conference on Circumstellar Habitable Zones, ed. L. R. Doyle (Menlo Park: Travis House), 229

Kovács, G., Zucker, S., \& Mazeh, T. 2002, A\&A, 391, 369

Lissauer, J. J., et al. 2011a, Nature, 470, 53

Lissauer, J. J., et al. 2011b, ApJ, submitted (arXiv:1102.0543)

Morton, T. D., \& Johnson, J. A. 2011, ApJ, submitted (arXiv:1101.5630)

Prša, A., et al. 2011, AJ, 141, 83

Queloz, D., et al. 2009, A\&A, 506, 303

Ragozzine, D., \& Holman, M. J. 2011, ApJ, submitted (arXiv:1006.3727)

Steffen, J. H., et al. 2010, ApJ, 725, 1226

Torres, G., et al. 2011, ApJ, 727, 24

Triaud, A. H. M. J., et al. 2010, A\&A, 524, 25

Winn, J. N., Fabrycky, D., Albrecht, S., \& Johnson, J. A. 2010, ApJ, 718, 145

Wright, J. T., Upadhyay, S., Marcy, G. W., Fischer, D. A., Ford, E. B., \& Johnson, J. A. 2009, ApJ, 693, 1084

Wu, H., et al. 2010, Proc. SPIE, 7740, 42

21 http://exoplanet.eu 